\title{
An Improved EMD with Second Generation Wavelet and Feature Extraction for Fault Diagnosis of Rotating Machinery
}

\author{
Fengli Wang* \\ College of Marine Engineering \\ Dalian Maritime University \\ Dalian, China \\ wangfl@dlmu.edu.cn \\ * Corresponding Author
}

\author{
Hui Xing \\ College of Marine Engineering \\ Dalian Maritime University \\ Dalian, China \\ xingcage@163.com
}

\section{Sihong Li}

Technology Development Center for Polymer Processing Engineering of Guangdong Province Guangdong Industry Technical College Guangdong, China

\begin{abstract}
Fault feature extraction is a challenge for fault diagnosis of rotating machinery. The vibration signals measured from rotating machinery are usually nonstationary and nonlinear. Especially, the useful fault characteristics are too weak to be identified at the early stage. In order to solve the problem, a novel method called improved empirical mode decomposition (EMD) with second generation wavelet for fault diagnosis of rotating machinery is proposed. According to the local characteristics of vibration signal and selecting the proper criterion of minimizing the squared error, an optimal predicting operator is constructed for a transforming sample, so that the second generation wavelet basis function is able to fit the local characteristics of the vibration signal. Using the selfadaptive second generation wavelet as the pre-filter to improve EMD decomposition results, EMD is further improved to increase the accuracy and effectiveness of the decomposition results. The proposed method is applied to analyze the rub-impact rotor experimental setup, and the results show that the proposed method is accurate and efficient, and is expected to be applied in engineering practice effectively.
\end{abstract}

Keywords-EMD; Fault Diagnosis; Feature Extraction; Second Generation Wavelet; Rotating Machinery

\section{INTRODUCTION}

Rotating machinery is one of the most common classes of mechanical equipment and plays an important role in industrial applications. With the development of technology, rotating machinery in industrial applications is becoming larger and more complex, such as gas turbine and aircraft engine. There exist faults in rotating machinery, for example, rub-impact, rotor imbalance, rotor misalignment, oil whirl or oil whip may occur. Research on fault diagnosis of rotating machinery is important to improve the performance and safety of rotating machinery.
Currently, many techniques of fault characteristic extraction have been proposed for the fault diagnosis of rotating machinery[1 4]. Most nondestructive damage identification methods can be categorized as either local or global damage identification techniques. The local damage identification techniques, such as ultrasonic methods and $\mathrm{X}$-ray methods, require that the vicinity of damage is known a priori and readily accessible for testing, which cannot be guaranteed for most cases in rotating machinery. Thus, as a global damage identification technique, the vibration-based damage identification method is developed to overcome these difficulties. The basic idea for vibrationbased damage identification is that the damage-induced changes in the physical properties will cause detectable changes in vibration performances. Therefore, it is intuitive that the damage can be identified by analyzing the changes in vibration features of the machinery structure. Traditional vibration signal processing techniques, including time-domain and frequency-domain analysis, are based on the assumption that the process generating signals is linear and stationary. However, due to nonstationary and nonlinearity of the vibration signals, the useful fault information is usually emerged in background noise. Especially, at the early fault stage, the fault features are too weak to be identified. They may result in false information once they are applied to the fault feature extraction of vibration signals.

To deal with non-stationary and nonlinearity of the vibration signals, several advanced time-frequency analysis techniques have been introduced and applied to fault diagnosis of rotating machinery[3 5]. Empirical mode decomposition (EMD) is one of the most powerful time-frequency analysis methods[5]. It is a self-adaptive time-frequency signal processing method. The original signal is decomposed into a series of intrinsic mode functions (IMFs), which are band limited and each IMFs component can be amplitude-modulated or frequency- 
modulated signal according to the signal characteristics with EMD[5]. It can well reveal the nonlinearity and nonstationary information of the vibration signal. It has been successfully applied to fault diagnosis of rotating machinery and structure health monitoring[6]. However, due to the problem of mode mixing, EMD cannot extract fault features accurately. To reduce the mode mixing, it is important to de-noise first before decomposing. Wavelet transform has been widely used for the signal de-noising. In the de-noising of traditional wavelet transform, the result of wavelet decomposing is related with wavelet basis function. Moreover, an inappropriate wavelet will over whelm the local characteristic of vibration signals, and lost some useful detail information of original signal. To overcome the above limitations, The lifting scheme is introduced to design self-adaptive wavelet functions for de-noising signal[7,8].

In this paper, researchers combine the advantages of EMD and second generation wavelet, and propose an improved EMD with second generation wavelet for fault diagnosis of rotating machinery. The second generation wavelet is used as the pre-filter to refine the vibration signal, and the improved EMD is used to extract the fault characteristics. The rest of this paper will be structured as follows. In Section II researchers will present a lifting scheme to construct self-adaptive second generation wavelets by the design of prediction operator and update operator. In Section III researchers will present the review of EMD and Hilbert transform. In Section IV researchers will describe our approach, explaining each step. In Section V researchers will present the experimental and practical validations. Finally, in Section VI researchers will give discussion and conclusion.

\section{SECOND GENERATION WAVELET DENOSING}

The lifting scheme is introduced by Sweldens as a powerful tool to construct biorthogonal wavelets in the spatial domain[7,8]. It provides a great deal of flexibility and freedom for constructing the biorthogonal wavelets. It can be used to construct self-adaptive second generation wavelets by designing the prediction operator and update operator, which consists in three main steps as follows.

(1) The original signal $\{X(k), k \in Z\}$ is split into two disjoint sets of samples. Usually, the signal $X(k)$ is split into even indexed samples and odd indexed samples. The splitting process can be described with the formulas as follows.

$$
\begin{aligned}
& X_{e}(k)=\{x(2 k), k \in Z\} . \\
& X_{o}(k)=\{x(2 k+1), k \in Z\} .
\end{aligned}
$$

(2) Using an update operator $U$ on the odd samples and adding the result to the even samples. The sequence $c(k)$ can be regarded as the approximation of the original signal $X(k)$.

$$
c(k)=X_{e}(k)+U\left[X_{o}(k)\right] .
$$

(3) We use the even samples to predict the odd one and apply a prediction operator $P$ on $X_{e}(k)$ to predict $X_{o}(k)$. The prediction error $d(k)$ should be possible to represent the detail of the original signal $X(k)$.

$$
d(k)=X_{o}(k)-P\left[X_{e}(k)\right] \text {. }
$$

Because the transform is only iterated on the low pass coefficients $c(k)$ which can be obtained by using an update operator, all coefficients $c(k)$ depend on the data. Then researchers use these low-pass coefficients to predict the odd samples, and obtain the high-pass coefficients $d(k)$. We use a linear update filter and select the predictor. Because the scaling vanishing moment and wavelet functions are related to both the number of approximation signal samples $(N)$ and the number of original signal samples( $\tilde{N}$ ). The scaling supported intervals and wavelet functions decreases with decreasing of both $N$ and $\tilde{N}$, which lead to fit the transient features of the original signal. By selecting the criterion of minimizing the squared error, researchers obtain an optimal prediction operator for a transforming sample so that the constructed wavelet function can fit the transient features of the original signal. The stages of lifting scheme wavelet transform is described as follows [7-9],

(1) Split the original signal $X(k)$ with the length of $L$ into even samples $X_{e}(k)$ and odd samples $X_{o}(k)$.

(2) The approximation signal $c(k)$ is calculated by using a one-point update filter.

$$
c(k)=\left(X_{e}(k)+X_{o}(k)\right) / 2
$$

(3) Select an optimal prediction operator: We design three different prediction operators as follows.

$$
N=1 \quad d(k)=X_{o}(k)-c(k)
$$

$$
\begin{aligned}
& \begin{array}{l}
N=3: d(k)=X_{o}(k)-[-c(k-1) / 8+c(k) \\
+c(k+1) / 8]
\end{array} \\
& \begin{aligned}
N=5: & d(k)=X_{o}(k)-\{3[c(k-2)-c(k+2)] / 128 \\
& +c(k)-11[c(k-1)-c(k+1)] / 64 \\
& -c(k+2)\} .
\end{aligned}
\end{aligned}
$$

Where, $N$ is the number of neighboring $c(k)$ while applying the prediction operator, $k=1 \sim L / 2$. We can select an optimal prediction operator for a transforming sample by minimizing the value of $[d(k)]^{2}$.

(4) The detail signal $d(k)$ is calculated by using the optimal prediction operator.

In the process of signal de-noising, researchers adopt the universal threshold. The wavelet coefficients can be calculated through soft-threshold with universal threshold at each level[10], 


$$
\text { thr }=\sqrt{2 \ln (n)} \cdot \frac{\operatorname{Med}(|d|)}{0.6745} .
$$

Where, $d=\{d(k)\}, n$ is the length of $d(k)$, and $\operatorname{Med}(\cdot)$ is median function.

\section{REVIEW OF EMD AND HILBERT TRANSFORM}

\section{A. EMD Method}

EMD method is developed by Huang and is able to decompose any signal into some intrinsic mode functions (IMFs), each of which is a function that satisfies the following two conditions [5]:(1) In the whole data set, the number of extrema and the number of zero-crossings must either equal or differ at most by one.(2) At any point, the mean value of the envelope defined by local maxima and the envelope defined by the local minima is zero.

An IMF represents a simple oscillatory mode imbedded in the signal. It is based on the simple assumption that a signal contains different simple IMFs. With the definition, any signal $\mathrm{x}(\mathrm{t})$ can be decomposed as follows[5]:

First, researchers find all the local maxima and local minima of the signal and use a two-tap time variant filter to get the local mean. Then, researchers use cubic spline to fit it as the local mean $m(t)$ and extract the detail $h(t)=x(t)-m(t)$. Regard $h(t)$ as new $x(t)$ and repeat the above steps until ${ }^{h(t)}$ satisfies the IMF conditions, then ${ }^{h(t)}$ is the first IMF $c_{1}(t)=h(t)$. Let the residual $r_{1}(t)=x(t)-h(t)$ be a new signal, repeat the above steps, and obtain the other orders IMFs. The decomposition process can be stopped when $r_{n}(t)$ becomes a monotonic function from, which no more IMF can be extracted. We finally obtain

$$
X(t)=\sum_{j=1}^{n} c_{j}(t)+r_{n}(t)
$$

Thus, researchers can decompose a signal into IMFs $c_{1}(t), c_{2}(t), \ldots, c_{n}(t)$, and a residue $r_{n}(t)$ which is the mean trend of $x(t)$. The IMFs $c_{1}(t), c_{2}(t), \ldots$, $c_{n}(t)$ include different frequency bands ranging from high to low. A more detailed explanation about EMD can be found in[5].

\section{B. The Hilbert transform of IMFs}

The template is used The EMD gives us a set of independent IMF components with meaningful instantaneous frequencies. Each of IMF components can be therefore treated as a signal where the Hilbert transform can be applied. With this definition, researchers can have an analytic signal as

$$
c_{j}(t)=\mathrm{a}_{\mathrm{j}}(\mathrm{t}) \mathrm{e}^{i \varphi_{\mathrm{j}}(\mathrm{t})} .
$$

After performing the Hilbert transform on each IMFs, the original signal $x(t)$ can be expressed as the real part $(R P)$ in the following form:

$$
X(t)=R P \sum_{j=1}^{n} \mathrm{a}_{\mathrm{j}}(\mathrm{t}) \mathrm{e}^{i \varphi_{\mathrm{j}}(\mathrm{t})} .
$$

Where

$$
\begin{aligned}
a_{j}(t) & =\sqrt{c_{j}^{2}(t)+H^{2}\left[c_{j}(t)\right]} . \\
\varphi_{j}(t) & =\arctan \frac{H\left[c_{j}(t)\right]}{c_{j}(t)} .
\end{aligned}
$$

\section{THE IMPROVED METHOD}

As mentioned above, the problem for EMD is that it is difficult to extract weak frequency characteristic components from the vibration signals. When there are faults in rotating machinery, the useful fault characteristic components usually have very little energy amplitude and are submerged among background noise, which contains wide frequency band noise and higher level macrostructural vibrations. It is very difficult to extract the useful fault characteristic components. The self-adaptive second generation wavelet is a good choice to solve the problem. The lifting scheme provides a great deal of flexibility and freedom for the construction of biorthogonal wavelets, and can be used to construct self-adaptive second generation wavelets by the design of prediction operator and update operator to match the given signal, and it can enhance the weak fault characteristic components. In this paper, researchers employ the self-adaptive second generation wavelet as the pre-filter bank to overcome the disadvantages of EMD and propose an improved EMD with second generation wavelet de-noising for fault diagnosis of rotating machinery. The procedures of the improved EMD algorithm are as follows:

(1) The lifting scheme is applied to construct a selfadaptive second generation wavelet for the original signal de-noising. The corresponding predictor and update operator are designed, and the predefined soft-thresholds is used to calculate the wavelet coefficients.

(2) After performing the wavelet de-noising, the original signal is reconstructed by using the second generation wavelet, and then can be decomposed into a small number of IMFs using EMD.

(3) Hilbert envelop spectrum analysis is applied to the sensitive IMFs and the fault features of the original signal is identified. 


\section{THE PROPOSED METHOD FOR EXPERIMENTS}

\section{A. Simulation Signals}

Here researchers consider a signal $X(t)$ with amplitude- modulated components,

$$
\begin{aligned}
x(t)= & 1.2(1+0.2 \sin (2 \pi \times 7.5 t)) \cos (2 \pi \times 30 t)+ \\
& 2.5 \sin (2 \pi \times 10 t)+0.25 \text { rand } n
\end{aligned}
$$

The total number of the simulation signal $n=1024$, the sampling frequency is $640 \mathrm{~Hz}$, and randn is a normally distributed random numbers vector with a mean of zero and a standard deviation of one. The time domain waveform of simulation signal is shown in Fig. 1. The simulation signal is analyzed with EMD method. The decomposition results are shown in Fig. 2. Due to the interference of noise, the mode mixture is occurring between IMFs. Thus, the decomposition results of EMD fail to represent the real characteristics of the simulation signal.

In order to solve the problem of mode mixing, the improved EMD method is applied to the simulation signal. First, the simulation signal is de-noised by using second generation wavelet, and then is decomposed by EMD and the results are shown in Fig. 3. It can be seen from Fig. 3 that the first IMF $c_{1}(t)$ has the obvious amplitudemodulated characteristic, and the second IMF $c_{2}(t)$ is sinusoidal component. Hilbert envelope analysis is applied to the first IMF $c_{1}(t)$ and the Hilbert envelope analysis of $c_{1}(t)$ are shown in Fig. 4 and 5. The Hilbert envelope amplitude of the IMF $c_{1}(t)$ shown in Fig. 4 is a slowly variational signal. From Fig. 5 there exists the obvious spectrum line which demonstrates the modulation frequency $7.5 \mathrm{~Hz}$. Therefore the proposed method can well reveal the modulation characteristics of the simulated signal.

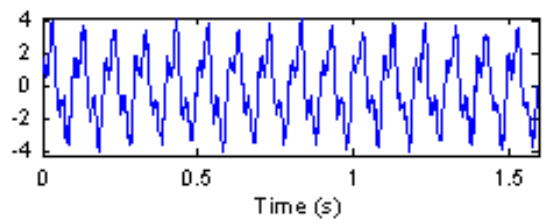

Figure 1. Time domain waveform of simulation signal.

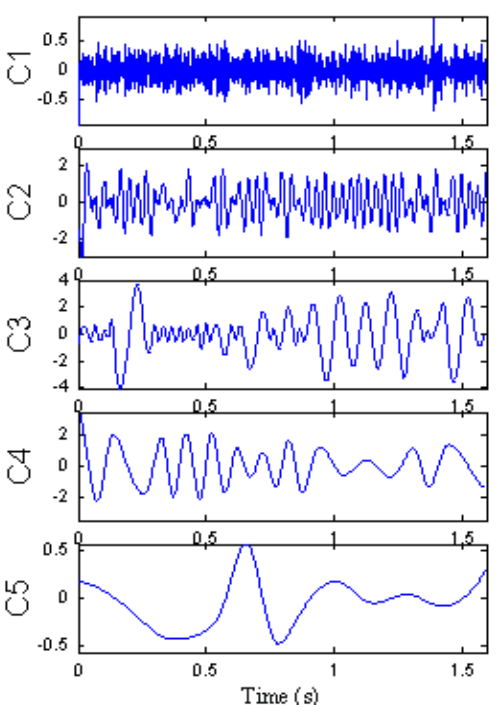

Figure 2. Decomposition results with EMD for simulation signal.
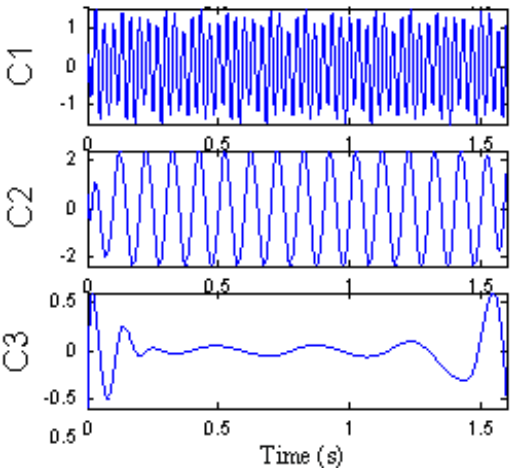

Figure 3. Decomposition results with improved EMD for simulation.

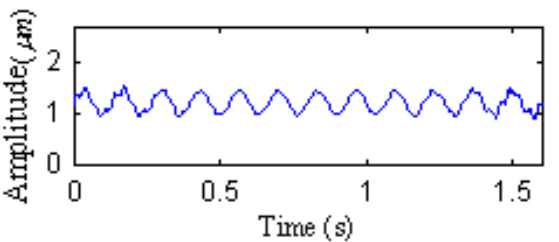

Figure 4. Hilbert envelope amplitude of the component $c_{1}(t)$.

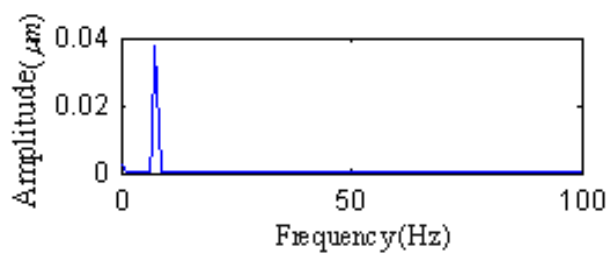

Figure 5. Hilbert envelope spectrum of the component $c_{1}(t)$.

\section{B. Experimental Evaluation}

Headings. In order to verify the validity of the proposed method, the local rub-impact fault occurs only in one position was conducted on a rotor experimental setup. Time domain waveform of the radial displacement vibration signal with local rub-impact fault picked up by the displacement sensor is shown in Fig. 6. The rotor 
rotating speed is $2820 \mathrm{r} / \mathrm{min}$ and the sampling frequency is $2560 \mathrm{~Hz}$.

To identify the rub-impact fault feature of rotor, firstly the second generation wavelet is applied to de-noise the vibration signal, then the de-noising signal is decomposed with EMD and the decomposition results are shown in Fig. 7. The first IMF $c_{1}(t)$ in Fig. 7 well reveals the amplitudemodulated characteristic. Hence, the first IMF $c_{1}(t)$ including the rub-impact information is just the rub-impact fault component. Hilbert envelope analysis is then applied to the first component $c_{1}(t)$. Fig. 8 illustrates that the Hilbert envelope amplitude function of the first component $c_{1}(t)$ is a slowly variational signal. From Fig. 9 it can be shown that there exists the obvious spectrum line which demonstrates the rotating frequency $47 \mathrm{~Hz}$. Therefore, the rub-impact fault feature of the rotor experimental setup can be identified in Fig. 9. The first and second decomposition components $c_{2}(t)$ and $c_{3}(t)$ are the background signals related to the rotating frequency.

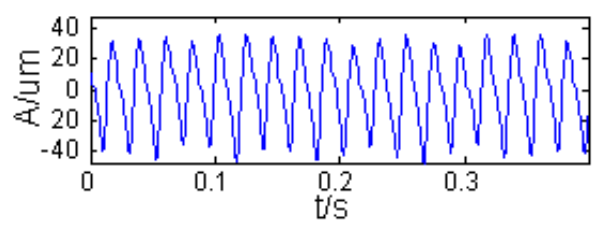

Figure 6. Time domain waveform of vibration signal.

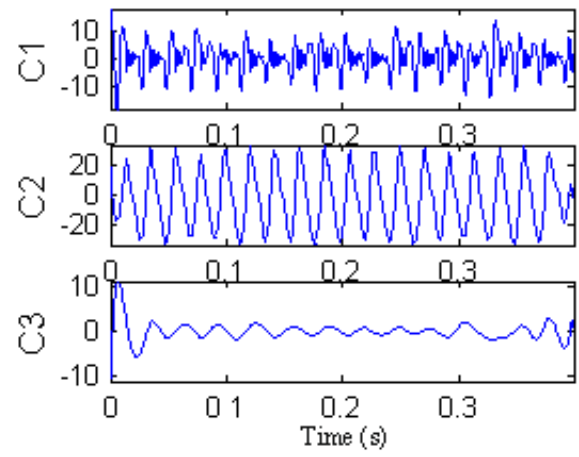

Figure 7. Decomposition results with improved EMD for vibration signal.

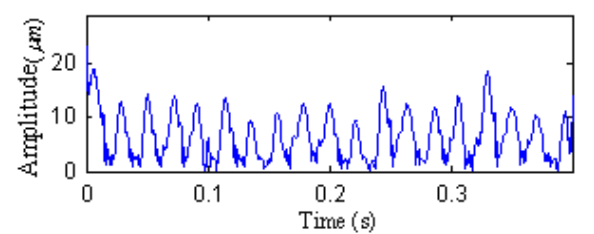

Figure 8. Hilbert envelope amplitude of the component $c_{1}(t)$.

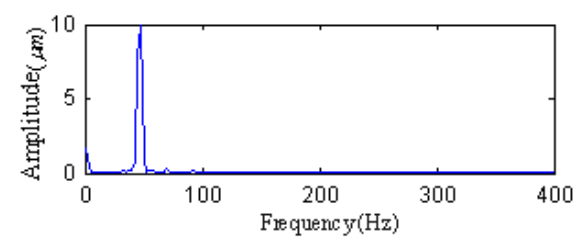

Figure 9. Hilbert envelope spectrum of the component $c_{1}(t)$

\section{CONCLUSIONS}

Targeting fault feature extraction for fault diagnosis of rotating machinery, a novel method called improved EMD with self-adaptive second generation wavelet is proposed in this paper.

(1) The lifting scheme provides a great deal of flexibility and freedom for constructing the biorthogonal wavelets. According to the local characteristics of vibration signal and selecting the proper criterion of minimizing the squared error, an optimal predicting operator is constructed for a transforming sample so that the second generation wavelet basis function is able to fit the local characteristics of the vibration signal. The selfadaptive second generation wavelet is used as the pre-filter, in order to improve EMD decomposition results.

(2) The proposed method is applied to analyze the rubimpact rotor experimental setup, and the results show that the proposed method is accurate and efficient to detect the rub-impact fault characteristics of rotating machinery, and is expected to be applied in engineering practice effectively.

\section{ACKNOWLEDGMENT}

The support for the Opening Project of Technology Development Center for Polymer Processing Engineering of Guangdong Province, Guangdong Industry Technical College (201503), National Nature Science Foundation of China (11272093) and Guangdong Province Higher Vocational Colleges\& Schools Pearl River Scholar Funded Scheme (2012) are gratefully acknowledged.

\section{REFERENCES}

[1] H. Zheng, Z. Li, X. Chen, and X. Chen, "Gear Fault Diagnosis Based on Continuous Wavelet Transform," Mech. Syst. Signal Process, vol. 16, 2002, pp. 447-457.

[2] M. Lal, R. Tiwari, "Multi-fault identification in simple rotorbearing-coupling systems based on forced response measurements," Mech. Mach. Theor., Vol.51,2012, pp. 87-109.

[3] S. Abbasion, A. Rafsanjani, A. Farshidianfar, et al., "Rolling element bearings multi-fault classification based on the wavelet denoising and support vector machine,"Mech. Syst. Signal Process, Vol.21, 2007, pp. 2933-2945.

[4] C. W. Bartelmus, R. Zimroz, "A new feature for monitoring the condition of gearboxes in non-stationary operating conditions," Mech. Syst. Signal Process,Vol.23, 2009, pp.1528-1534.

[5] N.E. Huang, Z. Shen, S.R. Long, et al, "The empirical mode decomposition and the Hilbert spectrum for nonlinear and nonstationary time series analysis,"Proc. of the Royal Society of London, Series A, Vol.459, 2003, pp. 2317-2345.

[6] Q. Gao, C. Duan, H. Fan, et al., "Rotating machine fault diagnosis using empirical mode decomposition," Mech. Syst. Signal Process, Vol. 22, 2008, pp.1072-1081.

[7] W. Sweldens, "The lifting scheme: A custom-design construction of biorthogonal wavele,"Appl. Comput. Harmon. Anal, Vol. 3, 1996, pp. 186-200.

[8] W. Sweldens, "The lifting scheme: A construction of second generation wavelet constructions," SIAM J. on Math. Anal., Vol. 29, 1997, pp. 511-546.

[9] R. L. Claypoole, M. D. Geoffrey, and W Sweldens, "Non- linear wavelet transforms for image coding via lifting,"IEEE Tran. on Image Proc., Vol. 12, 2003, pp. 1449-1459.

[10] D L Donoho, "De-noising by soft-thresholding". IEEE Tran. on Information Theory, Vol. 41, 1995,pp. 613-627. 